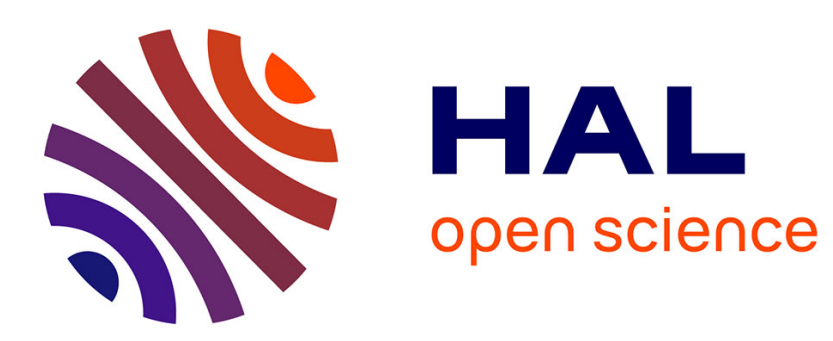

\title{
Overlooked problems inmanifold twins: twin misfit in zero-obliquity TLQS twinning and twin index calculation
}

\author{
Massimo Nespolo, Giovanni Ferraris
}

\section{To cite this version:}

Massimo Nespolo, Giovanni Ferraris. Overlooked problems inmanifold twins: twin misfit in zeroobliquity TLQS twinning and twin index calculation. Acta Crystallographica Section A : Foundations and Advances [2014-...], 2007, A63 (3), pp.278-286. 10.1107/S0108767307012135 . hal-00141588

\section{HAL Id: hal-00141588 \\ https://hal.science/hal-00141588}

Submitted on 13 Apr 2007

HAL is a multi-disciplinary open access archive for the deposit and dissemination of scientific research documents, whether they are published or not. The documents may come from teaching and research institutions in France or abroad, or from public or private research centers.
L'archive ouverte pluridisciplinaire HAL, est destinée au dépôt et à la diffusion de documents scientifiques de niveau recherche, publiés ou non, émanant des établissements d'enseignement et de recherche français ou étrangers, des laboratoires publics ou privés. 
Acta Crystallographica Section A

Foundations of

Crystallography

ISSN 0108-7673

Editor: D. Schwarzenbach

\section{Overlooked problems in manifold twins: twin misfit in zero-obliquity TLQS twinning and twin index calculation}

\section{Massimo Nespolo and Giovanni Ferraris}

Copyright (C) International Union of Crystallography

Author(s) of this paper may load this reprint on their own web site provided that this cover page is retained. Republication of this article or its storage in electronic databases or the like is not permitted without prior permission in writing from the IUCr. 
Acta Crystallographica Section A

Foundations of

Crystallography

ISSN 0108-7673

Received 15 February 2007

Accepted 14 March 2007

C 2007 International Union of Crystallography

Printed in Singapore - all rights reserved

\section{Overlooked problems in manifold twins: twin misfit in zero-obliquity TLQS twinning and twin index calculation}

\author{
Massimo Nespolo ${ }^{a *}$ and Giovanni Ferraris ${ }^{b}$ \\ aLCM3B UMR-CNRS 7036, Université Henri Poincaré Nancy I, BP 239, F-54506 Vandœuvre-lès- \\ Nancy CEDEX, France, and ${ }^{\mathbf{b}}$ Università di Torino, Dipartimento di Scienze Mineralogiche e \\ Petrologiche, Istituto di Geoscienze e Georisorse, CNR, Via Valperga Caluso 35, I-10125 Torino, \\ Italy. Correspondence e-mail: massimo.nespolo@Icm3b.uhp-nancy.fr
}

\begin{abstract}
It is shown that the twin index $n$ calculated, according to Friedel, as a function of the indices $(h k l)$ and $[u v w]$ of the lattice plane and lattice direction defining the cell of the twin lattice applies only to twofold twins, i.e. twins where the twin element is of order 2. For manifold twins, where the twin operation is a three-, four- or sixfold (direct or inverse) rotation, it is shown that the generalized formula becomes $n=N \Xi / \xi$, where $N$ is the number of lattice planes of the $(h k l)$ family passing within the cell of the twin lattice, $\Xi$ the two-dimensional coincidence index for a plane of the $(h k l)$ family and $\xi$ the number of planes out of $N$ of that family that are partially restored by the twin operation. The existence of twin lattice quasi-symmetry (TLQS) twins with zero-obliquity in manifold twins leads to the introduction of a new parameter as a general measure of the pseudo-symmetry of TLQS rotation twins: the twin misfit $\delta$, defined as the distance between the first nodes along the two shortest directions in the plane of $\mathbf{L}_{\mathrm{T}}$ (quasi-)perpendicular to the twin axes that are quasi-restored by the twin operation. Taking the example of staurolite twins, several inconsistencies in the treatment of manifold twins are pointed out.
\end{abstract}

\section{Introduction}

In this article, we use the same nomenclature and symbols described in our recent study on hybrid twins (Nespolo \& Ferraris, 2006). Here we give a short summary of the basic concepts of the reticular theory of twinning; more details can be found in the quoted article.

A twin is a heterogeneous crystalline edifice consisting of the oriented association of two or more homogeneous crystals (individuals). A twin operation is a crystallographic operation mapping the orientation of one individual on the orientation of another individual. A twin element is the lattice element of the individual (plane, row, centre) about which the twin operation is performed: Mallard's law (Friedel, 1926) states that a twin element is always a direct-lattice element, although it may sometimes be useful to use a reciprocal-lattice element instead, especially when unravelling the diffraction pattern of a twin, i.e. when the reciprocal lattice is inspected. The twin operation is a coincidence operation for the lattice of the individual ( $\mathbf{L}_{\text {ind }}$ hereafter) or a sublattice of it: the lattice nodes that are brought to coincidence are said to be 'restored' and define the twin lattice, $\mathbf{L}_{\mathrm{T}}$. This restoration may be exact or approximate and correspondingly one speaks of TLS (twin lattice symmetry) or TLQS (twin lattice quasi-symmetry), respectively (Donnay \& Donnay, 1974). Depending on the nature of the twin operation, twins are classified as reflection twins, rotation twins and inversion twins. In the latter case, $\mathbf{L}_{\text {ind }}$ coincides with $\mathbf{L}_{\mathrm{T}}$. For rotation or reflection twins, instead, this is not always the case and $\mathbf{L}_{T}$ can be a sublattice of $\mathbf{L}_{\text {ind }}$. The fraction of lattice nodes of $\mathbf{L}_{\mathrm{T}}$ restored by the twin operation corresponds to the ratio of the volume of primitive cells of $\mathbf{L}_{\mathrm{T}}$ and $\mathbf{L}_{\text {ind }}$.

The twin elements are (pseudo-)symmetry elements for the twin lattice $\mathbf{L}_{\mathrm{T}}$. A (pseudo-)symmetry plane ( $\left.h k l\right)$ is (quasi-) perpendicular to a lattice row $[u v w]$, and a (pseudo-)symmetry axis $[u v w]$ is (quasi-)perpendicular to a lattice plane $(h k l)$. The cell of $\mathbf{L}_{\mathrm{T}}$ is defined by the pair $[u v w]$ and $(h k l)$ and the twin index is easily computed in terms of the indices of these two elements, without the necessity of passing through the volume of the cells of $\mathbf{L}_{\text {ind }}$ and $\mathbf{L}_{\mathrm{T}}$ (Nespolo \& Ferraris, 2006). When $[u v w]$ and $(h k l)$ are not mutually perpendicular, the twin is of TLQS type; consequently, the symmetry of $\mathbf{L}_{\mathrm{T}}$ is close to a higher holohedry and the degree of pseudo-symmetry is normally given in terms of the obliquity, that is the angle between $[u v w]$ and the irrational direction perpendicular to $(h k l)$. These criteria were introduced by Friedel $(1904,1920$, 1926) and are routinely applied today. Hereafter, we show that they were implicitly limited to the case of twofold twins, i.e. 


\section{Table 1}

Classical classification of crystal twinning.

A finer classification is given in Nespolo \& Ferraris (2006). For twofold twins, the classification in terms of obliquity and of misfit (for the definition of the latter see \$3) coincides; however, for manifold twins the latter correctly assigns quasi-symmetry twins to pseudo-merohedry or reticular pseudo-merohedry even when zero obliquity would put them in a wrong category.

\begin{tabular}{llll}
\hline Twin index $n$ & Obliquity $\omega$ & Misfit $\delta$ & Revised Friedel classification \\
\hline \multirow{2}{*}{$=1$} & $=0$ & $=0$ & Merohedry \\
& $=0$ & $>0$ & Pseudo-merohedry \\
& $>0$ & $>0$ & \\
$>1$ & $=0$ & $=0$ & Reticular merohedry \\
& $=0$ & $>0$ & Reticular pseudo-merohedry \\
& $>0$ & $>0$ & \\
\hline
\end{tabular}

twins where the twin operation is of order 2. In this article, we prove that, for manifold twins, where the twin operation is a three-, four- or sixfold (direct or inverse) rotation [for details about definitions and classifications, see Nespolo (2004)], both the formula to compute the twin index in terms of $[u v w]$ and $(h k l)$ and the definition of TLQS in terms of the obliquity no longer apply. We propose a generalization of both criteria. The inconsistencies resulting from the uncritical application of Friedel's criteria to manifold twins are discussed by analysing the example of the classical twins in staurolite.

\section{Calculation of the twin index in manifold twins}

The classical classification of twins in terms of twin index and obliquity recognizes four categories (Table 1). Friedel's formula to calculate the twin index is (Grimmer \& Nespolo, 2006; see also Donnay \& Donnay, 1959, and Nespolo \& Ferraris, 2005)

$$
\begin{aligned}
& n=X / f \\
& X=|u h+v k+w l|,
\end{aligned}
$$

where $f=1,2$ or 4 depending on the Bravais-lattice type and the parities of $X, u, v, w, h, k, l$. ( $u, v, w$ have to be co-prime integers, ditto for $h, k, l$.) When $u, v, w, h, k, l$ are expressed in terms of a primitive basis of $\mathbf{L}_{\text {ind }}, f=1$ or 2 depending on whether $X$ is odd or even.

Let us consider the primitive cell of $\mathbf{L}_{\text {ind }} . X$ in equation (1) gives the number of lattice planes of the $(h k l)$ family between two neighbouring nodes along the $[u v w]$ direction. When the cell of $\mathbf{L}_{\text {ind }}$ is not primitive, the number of lattice planes between these two nodes may be $X / 2, X$ or $2 X$, depending on the parities of the indices (Table 2): we shall hereafter indicate this number as $N$.

If the twin operation is a twofold rotation about $[u v w]$, all nodes in the two planes of the $(h k l)$ family passing through two neighbouring nodes along the $[u v w]$ direction are restored (exactly in the case of TLS, approximately in the case of TLQS) because a two-dimensional mesh has at least symmetry 2. If $N$ is odd, all the other $N-1$ planes intersect the lattice row $[u v w]$ neither on a node nor midway between two
Table 2

Number $N$ of lattice planes of the family $(h k l)$ between two neighbouring nodes along the direction $[u v w]$ as a function of the lattice type, of the parity of the indices, and of the value $X=|u h+v k+w l| ; u, v, w$ are coprime integers, ditto for $h, k, l$.

\begin{tabular}{llll}
\hline Lattice type & Condition on $h, k, l$ & Condition on $u, v, w$ & $N$ \\
\hline$P$ & None & None & $X$ \\
$C$ & $h+k$ odd & $u+v$ and $w$ not both even & $2 X$ \\
& $h+k$ even & $u+v$ and $w$ both even & $X$ \\
& & $u+v$ and $w$ not both even & $X$ \\
& $h+l$ odd & $u+v$ and $w$ both even & $X / 2$ \\
$B$ & $h+l$ even & $u+w$ and $v$ not both even & $2 X$ \\
& & $u+w$ and $v$ both even & $X$ \\
& $k+l$ odd & $u+w$ and $v$ not both even & $X$ \\
& & $u+w$ and $v$ both even & $X / 2$ \\
$A$ & $k+l$ even & $v+w$ and $u$ not both even & $2 X$ \\
& & $v+w$ and $u$ both even & $X$ \\
& $h+k+l$ odd & $v+$ wand $u$ not both even & $X$ \\
& & $v+w$ and $u$ both even & $X / 2$ \\
$I$ & $h+k+l$ even & $u, v, w$ not all odd & $2 X$ \\
& & $u, v, w$ all odd & $X$ \\
& $h, k, l$ not all odd & $u, v, w$ not all odd & $X$ \\
& $h, k, l$ all odd & $u, v, w$ all odd & $X / 2$ \\
$F$ & $h, k, l$ not all odd & $u+v+w$ odd & $2 X$ \\
& $h, k, l$ all odd & & $X$ \\
& & & $X / 2$ \\
& & &
\end{tabular}

neighbouring nodes and therefore only one plane out of $N$ has all its nodes restored by the twin operation: the twin index is $N$. If instead $N$ is even, there is one plane of the same family which intersects the lattice row midway between two neighbouring nodes: the nodes on this plane are restored too. In this case, there are two planes out of $N$ having all their nodes restored by the twin operation and the twin index is $N / 2$.

When the twin operation is a rotation of higher degree about $[u v w]$, this simple criterion no longer applies, because in general the rotational symmetry of the two-dimensional mesh in the $(h k l)$ plane no longer coincides with that of the twin operation. The degree of restoration of lattice nodes must now take into account the two-dimensional coincidence index $\Xi$ for a plane of the family $(h k l)$, which defines a supermesh in $\mathbf{L}_{\mathrm{T}}$. Moreover, such a supermesh may exist in $\xi$ planes out of $N$, depending on where the intersection of the $[u v w]$ twin axis with the plane is located. The twin index is finally given by

$$
n=\frac{N \Xi}{\xi} \text {. }
$$

In the case of a twofold rotation, $\Xi=1$ and $\xi=1$ or 2 , which shows that equation (2) is a generalization of Friedel's equation (1) (remember that $N=2 X, X$ or $X / 2-$ see Table 2).

\section{Zero-obliquity TLQS: redefinition of pseudo- merohedry and reticular pseudo-merohedry}

Donnay \& Donnay (1974) introduced the categories of TLS and TLQS essentially with the purpose of distinguishing twins giving a diffraction pattern that shows 'either a single orientation of the reciprocal lattice or two (or more) distinct 
orientations having a common origin'. However, as shown in Table 1 therein, this distinction turns out to be simply a matter of separating zero-obliquity twins from those with non-zero obliquity, so that nowadays TLQS is used as a synonym for 'non-zero obliquity twinning'. This choice was somewhat unfortunate for at least two reasons:

1. the existence of 'two (or more) distinct orientations having a common origin' does not entirely rely on splitting of diffractions; twinning by reticular merohedry normally results in non-space-group absences which are among the strongest criteria to suspect the presence of twinning (see, for example, Nespolo \& Ferraris, 2003, and Ferraris et al., 2004);

2. a non-zero obliquity means that $\mathbf{L}_{T}$ is close to a higher holohedry - and this is at the origin of the term twin lattice quasi-symmetry; however, the opposite is not necessarily true, because a twin lattice can be pseudo-symmetric without the need for a twin operation to correspond to a non-zero obliquity: this is typically the case of manifold twins.

The second argument shows that the obliquity alone is not sufficient to differentiate between TLS and TLQS in the case of manifold twins, as was already pointed out by Santoro (1974) and later by Grimmer \& Nespolo (2006). Santoro (1974) introduced a measure of the deviation suffered by the twin lattice in crossing the boundary from one individual to the other. This measure consists in six parameters $\Delta_{i j}$ which all go to zero for zero obliquity only when the $\mathbf{L}_{T}$ twin lattice is not pseudo-symmetric. For zeroobliquity TLQS twins, which occur in manifold twins, at least one of the six parameters introduced by Santoro does not vanish. A different formalism, related to the study of grain boundaries, was introduced by Bonnet \& Durand (1975). They considered two transformation matrices: a linear part, representing the rotation necessary to complete the parallelism of two lattices starting from their misoriented alignment; and an affine part, which deforms one lattice to match the periodicity of the other one.

The two approaches above, despite the advantage of being quite general, give a measure of the lattice misorientation which is less immediate to read. Here we introduce a new single parameter, which not only gives the misorientation of the lattices but, differently from the other parameters introduced previously, for TLQS twins is also related to the twin index. The new parameter, which we call twin misfit $\delta$, is defined as the distance between the first nodes along the two shortest directions in the plane of $\mathbf{L}_{\mathrm{T}}$ (quasi-)perpendicular to the twin axis that are quasi-restored by the twin operation.

Let $u_{A} v_{A} w_{A}$ and $u_{B} v_{B} w_{B}$ be the coordinates in $\mathbf{L}_{\mathrm{T}}$ of two nodes quasi-restored by the twin operation. If $\mathbf{R}_{\varphi}$ is the rotation matrix representing in $\mathbf{L}_{\mathrm{T}}$ the crystallographic rotation by an angle $\varphi$ about the twin axis, the quasi-restoration is expressed by the equation

$$
\mathbf{R}_{\varphi}\left|u_{A} \quad v_{A} \quad w_{A}\right\rangle=\left|u_{A^{\prime}} \quad v_{A^{\prime}} \quad w_{A^{\prime}}\right\rangle \approx\left|u_{B} \quad v_{B} \quad w_{B}\right\rangle,
$$

where $\approx$ means quasi-equal. The twin misfit $\delta$ is then the distance between the coordinates $u_{A^{\prime}} v_{A^{\prime}} w_{A^{\prime}}$ and $u_{B} v_{B} w_{B}$ obtained as

$$
\begin{aligned}
& \delta=\langle\Delta u \quad \Delta v \quad \Delta w|\mathbf{G}| \Delta u \quad \Delta v \quad \Delta w\rangle^{1 / 2} \\
& \Delta u=u_{A^{\prime}}-u_{B} \\
& \Delta v=v_{A^{\prime}}-v_{B} \\
& \Delta w=w_{A^{\prime}}-v_{B}
\end{aligned}
$$

where $\mathbf{G}$ is the metric tensor of $\mathbf{L}_{\mathrm{T}}$. For TLQS, $u_{A^{\prime}} v_{A^{\prime}} w_{A^{\prime}}$ are in general irrational and can be obtained as follows. In general, the crystallographic basis of $\mathbf{L}_{\mathrm{T}}$ is not orthogonal; let $\mathbf{M}$ be the matrix transforming this crystallographic basis into an orthogonal basis. This transformation can be done via a classical Gram-Schmidt orthogonalization. Because the coordinates of a node are contravariant components, they are transformed by the inverse matrix, $\mathbf{M}^{-1}$. If 'cry' and 'or' indicate variables expressed in the crystallographic and the orthogonal basis respectively of $\mathbf{L}_{\mathrm{T}}$, the transformations are as follows:

$$
\begin{aligned}
& \mathbf{M}^{-1}\left|u_{A} \quad v_{A} \quad w_{A}\right\rangle_{\text {cry }}=\left|u_{A} \quad v_{A} \quad w_{A}\right\rangle_{\text {or }} \\
& \mathbf{R}_{\varphi}\left|u_{A} \quad v_{A} \quad w_{A}\right\rangle_{\text {or }}=\left|\begin{array}{lll}
u_{A^{\prime}} & v_{A^{\prime}} & w_{A^{\prime}}
\end{array}\right\rangle_{\text {or }}
\end{aligned}
$$

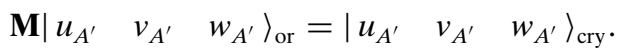

The rotation matrix $\mathbf{R}_{\varphi}$ is expressed in the orthogonal setting. For twofold twins, the above calculation simplifies drastically because there is a 1:1 relation between the obliquity and the twin misfit: the latter is the basis of an isosceles triangle whose vertex angle is $\omega$, so that $\delta=2 p \sin \omega$, where $p$ is the period of the shortest direction in the primitive mesh quasi-perpendicular to the twin axis, which forms the equal sides of the isosceles triangle (in the case of twofold rotation twins, all directions in this plane are quasi-restored).

Note that when the same direction may act as twin axis of different orders, giving different twins, the obliquity cannot differentiate these twins, whereas the twin misfit does, as will be shown for the [013] twins in staurolite.

The twin misfit is obviously zero for TLS twins. However, for TLQS twins the twin misfit is richer in information than the parameters previously introduced (Santoro, 1974; Bonnet \& Durand, 1975). Suppose two compounds $A$ and $B$ have the same space-group type but $A$ has larger lattice parameters than $B$. If both crystals undergo twinning according to the same twin law, they have the same twin index and the same obliquity, whereas the twin misfit $\delta_{A}$ is larger than $\delta_{B}$. Because the cell of $\mathbf{L}_{\mathrm{T}}$, and in particular the two-dimensional mesh in the contact plane, are also larger for $A$ than for $B$, the probability of occurrence of the twin is expected to be higher for $B$, all the other factors being constant. The other parameters proposed previously cannot differentiate the two cases.

Friedel's classification in Table 1 classifies twins by (reticular) pseudo-merohedry as having $\omega>0$. We have seen that for manifold twins this parameter is in general not sufficient to fully characterize these twins: we therefore propose to generalize the definition of twins by (reticular) pseudomerohedry as those twins having twin misfit $\delta>0$. 


\section{An instructive case study: the staurolite twins}

Smith (1968) concluded his study on the crystal structure of staurolite by saying that 'staurolite remains an enigma'. Although the structure and chemistry of staurolite are nowadays well known, we are going to show that the interpretation of its twins has been problematic up to now.

Well before a satisfactory structure solution of staurolite was achieved, this mineral attracted the interest of mineralogists because of its twins. The structure of staurolite was first solved by Náray-Szabó in 1929, who adopted the spacegroup type $\mathrm{Ccmm}$ suggested one year before by Cardoso (1928). This mineral is monoclinic, although its Bravais lattice at ambient conditions is metrically orthorhombic: the correct space group, of type $C 2 / \mathrm{m}$, was determined by Hurst et al. (1956). In the same year, Juurinen (1956) suggested $C 222_{1}$ as a possible space-group type of staurolite but later Náray-Szabó \& Sasvári (1958) refined the structure and confirmed Hurst et al.'s (1956) results. The chemical formula of staurolite was also a subject of discussion for many years, as shown, for example, by Griffen et al. (1982), Donnay \& Donnay (1983) and Alexander (1989).

Staurolite gives two penetration twins, known as the $90^{\circ}$ or 'Greek cross' twin and the $60^{\circ}$ or 'Saint Andrews cross' twin (Friedel, 1904, 1922). These twins are often reported as reflection twins on (031) and (232), respectively, but, as we are going to see, this interpretation is not correct. A third twin,

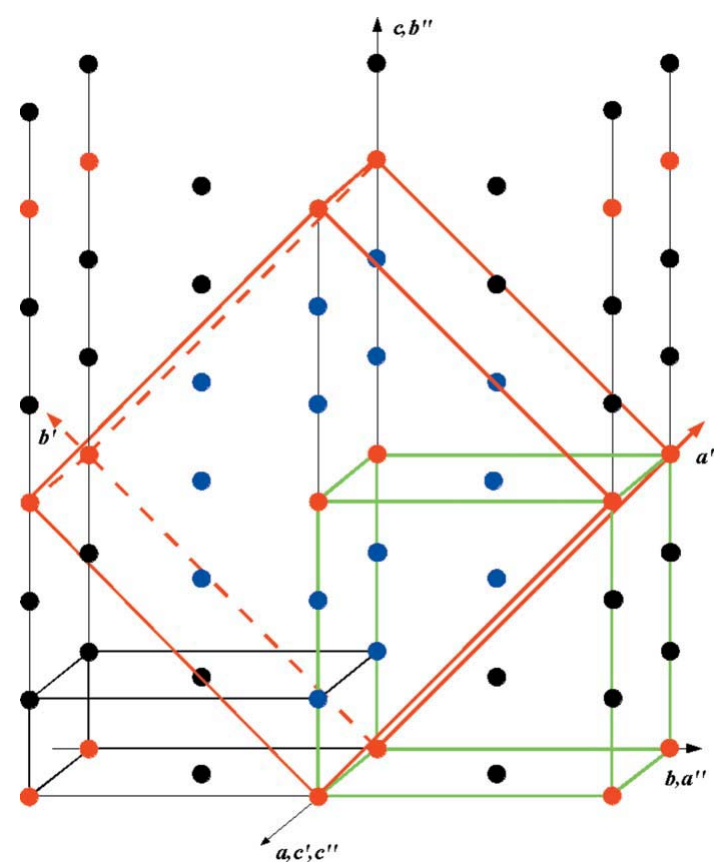

Figure 1

The pseudo-tetragonal $C$-centred (red) and the corresponding primitive (green) cell of the twin lattice for the Greek cross twin of staurolite. Red nodes define the cell of the twin lattice and are restored by the twin operations. Blue nodes are internal to the $\psi-t C$ cell, black nodes external to it. $\boldsymbol{a}, \boldsymbol{b}, \boldsymbol{c}$ are the basis vectors of the individual, $\boldsymbol{a}^{\prime}, \boldsymbol{b}^{\prime}, \boldsymbol{c}^{\prime}$ those of the $\psi-t C$ cell of the twin, $\boldsymbol{a}^{\prime \prime}, \boldsymbol{b}^{\prime \prime}, \boldsymbol{c}^{\prime \prime}$ those of the $\psi-t P$ cell of the twin. The cell of $\mathbf{L}_{\text {ind }}$ (black) is shown on the negative side of $\boldsymbol{b}$ for the sake of clarity. reported by Dana (1876) and quoted by Friedel (1904) as having [320] as twofold twin axis or (230) as twin plane, was only found once and never confirmed later (Hurst et al., 1956).

Both cross twins are twins by reticular pseudo-merohedry (TLQS) and can be rationalized in terms of a common lattice that is the pseudo-cubic primitive sublattice $(\psi-c P$; hereafter $\psi$ followed by the standard lattice-type symbol is used to indicate pseudo-symmetry) obtained from the $C$-centred cell known as 'Mallard's pseudo-cube' (Friedel, 1904). The latter is obtained from $\mathbf{L}_{\text {ind }}$ by the following transformation:

$$
\left\langle\left.\begin{array}{lll}
\boldsymbol{a} & \boldsymbol{b} & \boldsymbol{c}
\end{array}\right|_{\mathbf{L}_{\text {ind }}}\left[\begin{array}{ccc}
0 & 0 & 3 \\
1 & \frac{1}{1} & 0 \\
3 & 3 & 0
\end{array}\right]=\left\langle\left.\begin{array}{lll}
\boldsymbol{a} & \boldsymbol{b} & \boldsymbol{c}
\end{array}\right|_{\text {Mallard's pseudo-cube }}\right.\right.
$$

The supercell corresponding to Mallard's pseudo-cube has multiplicity 36 and is $C$-centred: this centring is not compatible with the cubic symmetry and therefore the lattice based on Mallard's pseudo cube is only pseudo-tetragonal $(\psi-t C)$, although it is metrically pseudo-cubic. Starting from the staurolite lattice parameters used by Hahn \& Klapper (2003), $a=7.871, b=16.620, c=5.656 \AA, \beta=90^{\circ}$, Mallard's pseudocube parameters are $a=23.752, b=23.752, c=23.613 \AA$, $\alpha=$ $90.00, \beta=90.00, \gamma=88.81^{\circ}$. The lattice based on Mallard's pseudo-cube is therefore $m C$ but $\psi-t C$ and metrically pseudocubic. Both twins can be obtained by using a symmetry element of the $\psi-c P$ sublattice as twin element, although for the Greek cross twin a smaller pseudo-tetragonal cell is sufficient to explain the twinning. As we are going to show, despite a number of studies on staurolite twins, some important mistakes still remain even in the most recent literature. In the following examples, when describing the choice of the axial setting to compute the twin misfit $\delta$, we will always take the twin axis as the $\boldsymbol{c}$ axis of $\mathbf{L}_{T}$ or of the orthogonal reference obtained from $\mathbf{L}_{T}$ by the orthogonalization process.

\subsection{Greek cross twin}

The $90^{\circ}$ twin is easier to explain: $\mathbf{L}_{\mathrm{T}}$ is the pseudo-tetragonal sublattice obtained from Mallard's pseudo-cube by taking 1/3 of its $c$ parameter, which thus coincides with the $a$ parameter of the individual; the cell parameters of $\mathbf{L}_{\mathrm{T}}$ are obtained by the transformation

$$
\left\langle\left.\begin{array}{lll}
\boldsymbol{a} & \boldsymbol{b} & \boldsymbol{c}
\end{array}\right|_{\mathbf{L}_{\text {ind }}}\left[\begin{array}{ccc}
0 & 0 & 1 \\
1 & \overline{1} & 0 \\
3 & 3 & 0
\end{array}\right]=\left\langle\left.\begin{array}{lll}
\boldsymbol{a} & \boldsymbol{b} & \boldsymbol{c}
\end{array}\right|_{\mathbf{L}_{\mathrm{T}}}\right.\right.
$$

The resulting cell of $\mathbf{L}_{T}$ is $\psi-t C$ and has multiplicity 12 ; the lattice parameters are $a=23.752, b=23.752, c=5.656 \AA, \alpha=$ $90.00, \beta=90.00, \gamma=88.81^{\circ}$. The twin index, 6 , corresponds to the value of the determinant of the transformation matrix because the two cells have the same multiplicity (both are $C$-centred). The setting of $\mathbf{L}_{T}$ is easily transformed to the conventional primitive $\psi$-tP cell, the transformation from the conventional cell of $\mathbf{L}_{\text {ind }}$ being 


$$
\left\langle\left.\begin{array}{lll}
\boldsymbol{a} & \boldsymbol{b} & \boldsymbol{c}
\end{array}\right|_{\mathbf{L}_{\text {ind }}}\left[\begin{array}{lll}
0 & 0 & 1 \\
1 & 0 & 0 \\
0 & 3 & 0
\end{array}\right]=\left\langle\left.\begin{array}{lll}
\boldsymbol{a} & \boldsymbol{b} & \boldsymbol{c}
\end{array}\right|_{\psi-t P} .\right.\right.
$$

Hurst et al. (1956) erroneously assigned twin index 3 instead of 6 to this twin. This error probably came from the fact that the transformation matrix from the $m C$ cell, metrically $o C$ ( $\mu$-oC hereafter), to the $\psi$ - $t P$ cell has determinant 3: the two cells however do not have the same multiplicity. The transformation matrix from $\mu-o C$ to $\psi-t C$ (same multiplicity) has determinant 6 , which coincides with the twin index.

The ratio of the point groups of the holohedries corresponding to $\mathbf{L}_{\mathrm{T}}$ and $\mathbf{L}_{\text {ind }}$ is $16 / 8=2$ (the lattice of staurolite is actually orthorhombic) and the twin is described by the dichromatic twin point group $4^{\prime} / \mathrm{m}^{2} / \mathrm{m}^{\prime} / \mathrm{m}^{\prime}$ (Curien \& Le Corre, 1958; Nespolo, 2004). The coset decomposition of the twin point group with respect to the point group of the individual gives two cosets, the first being the point group $\mathrm{mmm}$ of $\mathbf{L}_{\text {ind }}$ (elements indexed in the axial setting of the individual):

$$
\begin{aligned}
& 4^{\prime} / m 2 / m 2^{\prime} / m^{\prime} \\
&=\left\{1,2_{[010]}, \overline{1}_{1}, m_{[010]}, 2_{[001]}, 2_{[100]}, m_{[001]}, m_{[100]}\right\} \\
& \cup\left\{4_{[100]}^{+}, 2_{[01 \overline{1} 3]}, m_{[013]}, \overline{4}_{[100]}^{+}, 4_{[100]}^{-}, 2_{[013]}, m_{[013]}, \overline{4}_{[100]}^{-}\right\} .
\end{aligned}
$$

When doing a coset decomposition of a twin point group with respect to the point group of the individual, each coset represents a twin law and each operation in a coset is a possible twin operation. The Greek cross twin is obtainable by only one twin law. In the case of TLS twinning, all the operations in a coset are equivalent under the symmetry operations of the individual: any of them can be taken to represent the twin law, and it is then called coset representative. In the old textbooks, the coset representative was taken as (032) referred to the old morphological $c$ parameter, which is twice the structural parameter. After correction of the $c$ parameter, the coset representative was also amended to (031). In both cases, the twin was described as a reflection twin, despite several pieces of evidence since Friedel (1920) that staurolite twins are actually rotation twins. Furthermore, in our case $\mathbf{L}_{T}$ is only pseudo-tetragonal and twinning is thus of TLQS type: the eight operations in each coset in equation (8) are only approximately equivalent; besides, each pair of twin operations related by the inversion centre of the crystal form a pair of non-equivalent corresponding twins (Friedel, 1926), also known as reciprocal twins (Mügge, 1898).

The above analysis can be found also in Hahn \& Klapper (2003), which is however affected by a problem in the calculation of the twin index, resulting in an inconsistent classification of the twin operations. Four of the twin operations in the coset are fourfold (direct or inverse) rotations about the [100] direction, which is perpendicular to the (100) lattice plane. The obliquity is zero but this is clearly a case of TLQS twinning, because $\mathbf{L}_{\mathrm{T}}$ is only pseudo-tetragonal; in fact, $a=b=$ $23.752 \AA$ but $\gamma=88.81^{\circ}$ [a similar case of twinning in leucite was described by Grimmer \& Nespolo (2006)]. If one simply applies Friedel's formula to these operations, one would get twin index 1, whereas the same formula applied to the other four operations gives the correct value of 6 . Evidently, two operations in the same coset cannot correspond to different twin indices because they would no longer be equivalent under the action of the subgroup in terms of which the group of $\mathbf{L}_{\mathrm{T}}$ has been decomposed. Nevertheless, Table 3.3.9.1 in Hahn \& Klapper (2003) divides the eight possible twin operations into two groups, one containing the fourfold operations and corresponding to a 'twin index 1', the other corresponding to twofold operations and to twin index 6. Fig. 1 shows a portion of $\mathbf{L}_{\text {ind }}$, where the $\psi$ - $t C$ (red) and $\psi$ - $t P$ (green) cells have been marked. Red nodes are restored by the twin operation (they are the nodes at the corners of the cell of $\mathbf{L}_{\mathrm{T}}$ and, for the case of the $\psi-t C$ cell, at the centre of the $C$ face); blue nodes are internal to the $\psi$ - $t C$ cell of $\mathbf{L}_{\mathrm{T}}$ but not restored by the twin operations; black nodes are external to the $\psi-t C$ cell of $\mathbf{L}_{\mathrm{T}}$. A rapid glance at the figure shows that the $\psi$ - $t C$ cell contains 12 nodes, of which two are restored (red ones): the twin index is 6 , as expected. The $\psi$ - $t P$ cell of $\mathbf{L}_{\mathrm{T}}$ contains red nodes only at the corners, namely one out of six, giving once again twin index 6 . If we now look at the result of a fourfold rotation about [100] of the individual (that is, about [001] of the twin), we see that $N=2 X=2$ (Table 2), one node (the red one) out of three is restored by the twin operation for the lattice planes of the (100) family passing through a lattice node on the [100] direction (thus $\Xi=3$ ) and none for the other planes of the same family, i.e. the partial restoration is realized for one plane out of $N=2$, so that $\xi=1$. Equation (2) gives the twin index: $3 \times 2 / 1=6$, as expected. The obliquity is $\omega=0^{\circ}$ despite the TLQS nature of this twin. The twin operation does not bring the 100 node of the twin lattice to coincide exactly with the 010 node but slightly away from it because of the $88.81^{\circ}$ angle between the $[100]_{\mathbf{L}_{\mathrm{T}}}$ and the $[010]_{\mathbf{L}_{\mathrm{T}}}$ directions. The distance between the two nodes, which represents the twin misfit, is $\delta=23.752 \sin (90-88.81)=0.493 \AA$.

Hurst et al. (1956) gave three possible twin operations in the Greek cross twin: $4_{[100]}, 2_{[013]}$ and $(031)=m_{[013]}$. The three operations above are also those given by Friedel (1922) once the correct lattice parameters are chosen (Donnay \& Donnay, 1983). Friedel (1922) chose as twin operation $4_{[100]}$, not because of a 'lower twin index', as suggested by Hahn \& Klapper (2003) but because, despite the scarcity of samples, he could measure the angle between the faces $g^{1}$ - corresponding to the (010) faces in the Haüy-Lévy notation - and found that the best accord was for the values computed for this twin operation. Hurst et al. (1956) performed an X-ray precession study on three Greek cross twins and found that in two cases Friedel's choice was correct, while in the third case the twin operation was $2_{[013]}$ : this third case corresponds to $\omega=1.19^{\circ}$. The shortest lattice direction in the (031) plane, quasiperpendicular to [013], is the $a$ axis of $\mathbf{L}_{\text {ind }}$ but this direction is exactly restored by the twin operation: in fact, $\gamma\left(\mathbf{L}_{\text {ind }}\right)=90^{\circ}$, which implies that also $\alpha\left(\mathbf{L}_{\mathrm{T}}\right)=90^{\circ}$. The second shortest direction in the (031) plane is [013], $p=23.752 \AA$ and the obliquity is $1.19^{\circ}$. It follows that $\delta=2 \times 23.752 \sin (1.19)=$ $0.987 \AA$. All the three samples investigated are rotation twins and not reflection twins: Hurst et al. (1956) pointed out that a 
Table 3

Coset decomposition of $m \overline{3} \mathrm{~m}$ in terms of $4 / \mathrm{mmm} ; \mu$-oC: axial setting of the individual (metrically $o C) ; \psi-c P$ : axial setting of the twin (pseudo-cubic).

\begin{tabular}{|c|c|c|c|c|c|}
\hline \multicolumn{2}{|c|}{$4 / m m m$} & \multicolumn{2}{|c|}{ First coset } & \multicolumn{2}{|c|}{ Second coset } \\
\hline$\mu-o C$ & $\psi-c P$ & $\mu-o C$ & $\psi-c P$ & $\mu-o C$ & $\psi-c P$ \\
\hline 1 & 1 & $4_{[0 \overline{1} 3]}^{+}$ & $4_{[010]}^{+}$ & $4_{[013]}^{+}$ & $4_{[100]}^{+}$ \\
\hline $2_{[010]}$ & $2_{[1 \overline{1} 0]}$ & $3_{[320]}^{t}$ & $3_{[1 \overline{1} \overline{1}]}^{+}$ & $3_{[\overline{3} 20]}^{-}$ & $3_{[11 \overline{1} \overline{1}]}^{-}$ \\
\hline$\overline{1}$ & $\overline{1}$ & $\overline{4}_{[0 \overline{1} 3]}^{+}$ & $\overline{4}_{[010]}^{+}$ & $\overline{4}_{[013]}^{+}$ & $\overline{4}_{[100]}^{+}$ \\
\hline$m_{[010]}$ & $m_{[1 \overline{1} 0]}$ & $\overline{3}_{[320]}^{+}$ & $\overline{3}_{[1 \overline{1} \overline{1}]}^{+}$ & $\overline{3}_{[\overline{3} 20]}^{-}$ & $\overline{3}_{[\overline{1} \overline{1} \overline{1}]}^{-}$ \\
\hline $2_{[001]}$ & $2_{[110]}$ & $3_{[10 \overline{2}]}^{+}$ & $3_{[1 \overline{1} 1]}^{t}$ & $3_{[102]}^{-}$ & $3_{[111]}^{-}$ \\
\hline $2_{[100]}$ & $2_{[001]}$ & $2[313]$ & $2_{[101]}$ & $2_{[31 \overline{3}]}$ & $2_{[01 \overline{1}]}$ \\
\hline$m_{[001]}$ & $m_{[110]}$ & $\overline{3}_{[10 \overline{2}]}^{+}$ & $\overline{3}_{[\overline{111}]}^{t}$ & $\overline{3}_{[102]}^{-}$ & $\overline{3}_{[111]}^{-}$ \\
\hline$m_{[100]}$ & $m_{[001]}$ & $m_{[313]}$ & $m_{[101]}$ & $m_{[31 \overline{3}]}$ & $m_{[01 \overline{1}]}$ \\
\hline $4_{[100]}^{+}$ & $4_{[001]}^{+}$ & $3_{[102]}^{+}$ & $3_{[111]}^{+}$ & $3_{[320]}^{-}$ & $3_{[\overline{\overline{1}} 1 \overline{1}]}$ \\
\hline $4_{[100]}^{-}$ & $4_{[001]}^{-}$ & $3_{[320]}^{+}$ & $3_{[11 \overline{1}]}^{t}$ & $3_{[10 \overline{2}]}^{-}$ & $3_{[\overline{1} \overline{1} 1]}$ \\
\hline$\overline{4}_{[100]}^{+}$ & $\overline{4}_{[001]}^{+}$ & $\overline{3}_{[102]}^{+}$ & $\overline{3}_{[111]}^{+}$ & $\overline{3}_{[320]}^{-}$ & $\overline{3}_{[\overline{1} 11 \overline{1}]}$ \\
\hline$\overline{4}_{[100]}^{-}$ & $\overline{4}_{[001]}^{-}$ & $\overline{3}_{[320]}^{+}$ & $\overline{3}_{[\overline{111}]}^{+}$ & $\overline{3}_{[10 \overline{2}]}^{-}$ & $\overline{3}_{[\overline{1} \overline{1} 1]}$ \\
\hline $2_{[013]}$ & $2_{[100]}$ & $2_{[\overline{3} 13]}$ & $2_{[\overline{1} 01]}$ & $4_{[013]}^{-}$ & $4_{[100]}^{-}$ \\
\hline$m_{[013]}$ & $m_{[100]}$ & $m_{[\overline{3} 13]}$ & $m_{[\overline{1} 01]}$ & $\overline{4}_{[013]}^{-}$ & $\overline{4}_{[100]}^{-}$ \\
\hline $2_{[0 \overline{1} 3]}$ & $2_{[010]}$ & $4_{[0 \overline{1} 3]}^{-}$ & $4_{[010]}^{-}$ & $2[3 \overline{1} 3]$ & $2_{[011]}$ \\
\hline$m_{[0 \overline{1} 3]}$ & $m_{[010]}$ & $\overline{4}_{[0 \overline{1} 3]}^{-}$ & $\overline{4}_{[010]}^{-}$ & $m_{[3 \overline{1} 3]}$ & $m_{[011]}$ \\
\hline
\end{tabular}

sponding to 16 different operations that would be strictly equivalent in the case of TLS, whereas they are only approximately equivalent in TLQS.

The cell of $\mathbf{L}_{\mathrm{T}}$ for the Saint Andrews cross twin can be easily obtained from the $\psi$ - $t C$ (red) cell in Fig. 1, by taking three times the period along the monoclinic $a$ axis (the $c$ axis of $\mathbf{L}_{\mathrm{T}}$ ) and by drawing as blue the node at the centre of the $C$ face. The coset decomposition is given in Table 3 in terms of both the $\mu-o C$ cell of $\mathbf{L}_{\text {ind }}$ and the $\psi-c P$ cell of $\mathbf{L}_{\mathrm{T}}$, to facilitate the comparison. The twin index is in all cases 12 , but in the literature we find different values; we shall briefly explain the origin of these mistakes.

The operations in Table 3 are the same as those given by Hurst et al. (1956), who did not classify them in terms of twin laws. Apart from some indexing differ-

reflection twin cannot be a penetration twin because the composition surface cannot be plane.

Because the structure of staurolite is actually monoclinic, further twinning by metric merohedry (Nespolo \& Ferraris, $2000)$ is also possible, which corresponds to the dichromatic point group $2^{\prime} / m^{\prime} 2 / m 2^{\prime} / m^{\prime}$, with coset decomposition

$2^{\prime} / m^{\prime} 2 / m 2^{\prime} / m^{\prime}=\left\{1,2_{[010]}, \overline{1}, m_{[010]}\right\} \cup\left\{2_{[001]}, 2_{[100]}, m_{[001]}, m_{[100]}\right\}$.

Being a twin by (metric) merohedry, this one could coexist with the cross twin without being detected by morphological analysis. The geometry of the diffraction pattern would not reveal its presence either, unless significant deviation of the $\beta$ angle from $90^{\circ}$ exists. To our knowledge, this additional twinning has not been confirmed so far.

\subsection{Saint Andrews cross twin}

For the Saint Andrews cross twin, $\mathbf{L}_{\mathrm{T}}$ is the $\psi$-cP sublattice of the $\psi-t C$ lattice defined by Mallard's pseudo-cube. In fact, the latter is metrically pseudo-cubic but the $C$-centring is not compatible with a cubic (pseudo-)symmetry. Hahn \& Klapper (2003, p. 424) stated that, because of this centring, 'the $60^{\circ}$ cross cannot be explained by the lattice construction of the pseudo-cube'. Actually, this twin is explained by taking the $\psi$ - $c P$ sublattice of Mallard's pseudo-cube obtained by ignoring the $C$-centring vector. In dealing with twinning, to take a sublattice means that the nodes being neglected are not restored by the twin operation. The twin laws are found by decomposing the cubic holohedry (pseudo-symmetry of the twin lattice of the Saint Andrews cross twin) in terms of the tetragonal holohedry (pseudo-symmetry of the twin lattice of the Greek cross twin). The ratio of the order of the point groups corresponding to the cubic and the tetragonal holohedries is $48 / 16=3$ and there are thus two possible twin laws (the third coset being the tetragonal holohedry), each corre- ence because at those times the cell of staurolite was given with twice the correct $c$ parameter, five of these operations were given also by Friedel (1922), namely: $3_{[102]}, 3_{[320]}, 2_{[313]}$, (231) and $4_{[013]}$. When indexed with respect to the axial setting of $\psi-c P$, these become, respectively: $3_{[111]}, 3_{[11 \overline{1}]}, 2_{[101]}$, (101) and $4_{[100]}$. The first four operations belong to the first coset, the last one to the second coset. Hurst et al. (1956) confirmed $3_{[102]}$ in one case and $2_{[313]}$ in two other cases. They stated that the pseudo-cubic cell has multiplicity 18 , which corresponds to the determinant of the transformation matrix in equation (5). However, the cell of the individual is $C$-centred and thus the cell of $\mathbf{L}_{\mathrm{T}}$, which is 18 times larger, has multiplicity 36 . Because of this mistake, Hurst et al. (1956) assigned twin index 6 instead of 12 to this twin.

Hahn \& Klapper (2003) (Table 3.3.9.1) assigned four different values to the twin index of the Saint Andrews cross twin: 3 for $3_{[102]}, 9$ for $3_{[320]}, 6$ for $4_{[013]}$, and 12 for $2_{[313]}$ and (231). ${ }^{\mathbf{1}}$ These inconsistencies come once again from the direct application of Friedel's formula (1): in fact, the correct value of 12 is found when this formula is applied to binary operations, like $2_{[313]}$ and (231), which are mutually quasi-perpendicular. In the other cases, the calculation should be performed with the formula in equation (2).

(i) The direction [102] is quasi-perpendicular to (101), so that $N=2 X=6$ (Table 2). The directions defining the primitive two-dimensional mesh in the (101) plane are [010] and [10̄1], $90^{\circ}$ apart, whose parameters are 16.620 and $9.619 \AA$, namely $a \simeq b \times 3^{1 / 2}$, the classical orthohexagonal relation. In this plane, we can therefore find a pseudo-hexagonal sublattice with coincidence index $\Xi=2$ : every second node is restored by the twin operation. Had the mesh been centred, the whole

\footnotetext{
${ }^{1}$ Hahn \& Klapper included also (031) among the twin elements of the Saint Andrews twin. This is however a twin plane for the Greek cross twin and a symmetry plane for the $\psi-t P$ sublattice.
} 


\section{research papers}

two-dimensional lattice would be pseudo-hexagonal [other examples of the same kind are in aragonite, $\mathrm{NH}_{4} \mathrm{LiSO}_{4}$ and compounds of the $\mathrm{K}_{2} \mathrm{SO}_{4}$ type, see Hahn \& Klapper (2003, p. 424)]. Only one plane of the family (101) behaves in the same way when a threefold rotation is applied about the [102] direction, so that $\xi=1$. The twin index is therefore $6 \times 2 / 1=$ 12 , as expected. A geometrical interpretation is easily obtained by taking three pseudo-tetragonal cells in Fig. 1: the

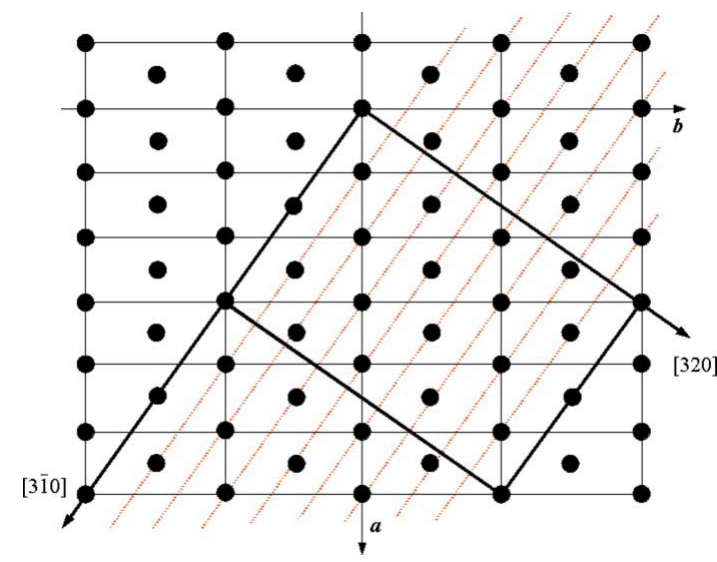

Figure 2

The (001) plane of staurolite with the projection of the cell of the twin lattice corresponding to the $[320] /(130)$ pair marked in bold. [310] and [320] are two of the directions defining the cell of $\mathbf{L}_{\mathrm{T}}$, the third one being [001], the direction of the projection. The red dotted rows are the trace of the planes of the family (130) passing within the cell of $\mathbf{L}_{\mathrm{T}}$ : there are nine planes of this family from the origin to the first node along the [320] direction.



Figure 3

The (130) plane of staurolite with its pseudo-hexagonal mesh of multiplicity 12 shown in red. The axes of this mesh are along the [313] and [313] directions of staurolite (33.492 $\AA$ ), the inter-axial angle being $119.12^{\circ}$. first node along the [102] direction on the corner of Mallard's pseudo-cube is 306 . Besides the nodes at the corners, two other nodes on the direction [102], namely 102 and 204, are also restored, leading to the twin index of $36 / 3=12$.

(ii) The direction [320] is quasi-perpendicular to (130). Hahn \& Klapper (2003) attributed twin index 9 to the twin produced by a threefold rotation about the [320] direction. As shown in Fig. 2, nine is the number $N=X$ (Table 2) of lattice planes of the family (130) from the origin to the first node along the direction [320]: it would coincide with the twin index only if the twin operation were a twofold rotation. Fig. 3 shows that in the (130) plane there is a pseudo-hexagonal mesh of multiplicity 12 and 12 is also the coincidence index $\Xi$ : the nodes defining this mesh (red in Fig. 3) are quasi-restored by the twin operation, the others are not. One can show that the same holds for all the nine planes: in Fig. 3, the lattice rows parallel to the $c$ axis can be divided in two types which alternate regularly: one has one node out of six (quasi-)restored by the twin operation, the other has no node restored at all, which eventually gives $\Xi=12$. The same holds for the next plane: the row $[10 l]$ has (quasi-)restored the nodes with $l=2(\bmod 6)$, the row $[4 \overline{1} l]$ has (quasi-)restored the nodes with $l=5(\bmod 6)$, the lattice row passing through the node at $2.5 a$ and $-0.5 b$ has none of its nodes restored. This finally leads to $\xi=9$, so that the twin index is $9 \times 12 / 9=12$, as expected.

(iii) The direction [013] is the same as that responsible for one of the Greek cross twins when it acts as twofold axis; when instead it acts as a fourfold axis, it gives one of the Saint Andrews cross twins. Hahn \& Klapper (2003) attributed twin



Figure 4

(100) projection of the staurolite lattice showing in red the directions [013] and [013] defining the cell of the twin lattice. Dotted red lines are the traces of the 11 planes of the family (031) between the origin and the first node along the [013] direction. The numbers in colour are the $u$ coordinate, expressed in the axial setting of the individual, of the lattice nodes inside the cell of the twin lattice restored by the operations $4_{[013]}^{+}$ (green), $4_{[013]}^{-}$(blue) and $2_{[013]}$ (red). 
index 6 instead of 12: the result is correct for the Greek cross twin, not for the Saint Andrews cross twin. The number of lattice planes of the family (031) between the origin and the first node along the [013] direction is $N=2 X=12$ - see Table 2 and Fig. 4. The coincidence index of the (031) plane for a fourfold rotation is $\Xi=3$, but only three planes out of the twelve are partially restored by the twin operation $(\xi=3)$ : besides the plane at the origin, also the plane containing nodes 002 and 004 is restored (indexing with respect to the axial setting of $\mathbf{L}_{\text {ind }}$ ). In fact, a fourfold rotation about [013] restores, besides the node at the origin, the nodes 202 and 104 (positive rotation) or 102 and 204 (negative rotation): these are the lattice nodes that, after application of the twin operation, have their $u v w$ indices transformed to integer $w$ and integer or half-integer $u$ and $v$. The twin index is thus $12 \times 3 / 3=12$, as expected.

It is particularly instructive to compare the measure of the pseudo-symmetry of $\mathbf{L}_{\mathrm{T}}$ in the cases above by means of the obliquity $\omega$ and of the twin misfit $\delta$.

(i) For $3_{[102]}$, the lattice plane quasi-perpendicular to the twin axis is (101) and the pseudo-hexagonal mesh therein is defined by the directions [202] and [111] forming an angle of $120.25^{\circ}$. The parameters of $\mathbf{L}_{\mathrm{T}}$ are $\mathbf{a}_{\mathrm{T}}=[11 \overline{1}]_{\mathbf{L}_{\text {ind }}}(19.240 \AA), \mathbf{b}_{\mathrm{T}}=$ $[\overline{2} 02]_{\mathbf{L}_{\text {ind }}}(19.385 \AA), \mathbf{c}_{\mathrm{T}}=[102]_{\mathbf{L}_{\text {ind }}}(13.781 \AA), \alpha_{\mathrm{T}}=89.13, \beta_{\mathrm{T}}=$ $90.44, \gamma_{\mathrm{T}}=120.25^{\circ}$. The obliquity is $\omega=1.49^{\circ}$ and the twin misfit is $\delta=2.548 \AA$.

(ii) For $3_{[320]}$, the lattice plane quasi-perpendicular to the twin axis is (101) and the pseudo-hexagonal mesh therein is defined by the directions [ $\overline{3} 13]$ and [313]. The parameters of $\mathbf{L}_{T}$ are $\mathbf{a}_{\mathrm{T}}=[\overline{3} 13]_{\mathbf{L}_{\text {ing }}}(33.492 \AA), \mathbf{b}_{\mathrm{T}}=[313]_{\mathbf{L}_{\text {ind }}}(33.492 \AA), \mathbf{c}_{\mathrm{T}}=$ $[320]_{\mathbf{L}_{\text {ind }}}(40.773 \mathrm{~A}), \alpha_{\mathrm{T}}=89.79, \beta_{\mathrm{T}}=90.22, \gamma_{\mathrm{T}}=119.12^{\circ}$. The obliquity is $\omega=0.37^{\circ}$ and the twin misfit is $\delta=4.331 \AA$.

(iii) For $4_{[013]}$, the lattice plane quasi-perpendicular to the twin axis is (031) and the pseudo-tetragonal mesh therein is defined by the directions [013] and [013]. The parameters of $\mathbf{L}_{\mathrm{T}}$ are $\mathbf{a}_{\mathrm{T}}=3 \times[100]_{\mathbf{L}_{\text {ind }}}(23.613 \AA), \mathbf{b}_{\mathrm{T}}=[013]_{\mathbf{L}_{\text {ind }}}(23.752 \AA), \mathbf{c}_{\mathrm{T}}=$ $[0 \overline{13}]_{\mathbf{L}_{\text {ind }}}(23.752 \AA), \alpha_{\mathrm{T}}=88.81, \beta_{\mathrm{T}}=90, \gamma_{\mathrm{T}}=90^{\circ}$. The obliquity is $\omega=1.19^{\circ}$ and the twin misfit is $\delta=0.493 \AA$.

(iv) For $2_{[313]}$, the lattice plane quasi-perpendicular to the twin axis is (231). The shortest direction in this plane is [102] $(13.781 \AA)$, the obliquity is $1.30^{\circ}$ and the twin misfit is $\delta=$ $2 \times 13.781 \sin (1.30)=0.625 \AA$.

These four twins correspond to similar obliquities but quite different twin misfits. Hurst et al. (1956) have confirmed the two twins corresponding to intermediate misfits $(0.625$ and $2.548 \AA$ ). We would expect to find the one with the smallest misfit $(0.493 \AA)$ but probably not the last one, $3_{[320]}$, because of the very large twin misfit.

It should also be emphasized, as mentioned above, that the twin misfit depends on the type of twin operation about the same direction of $\mathbf{L}_{\text {ind }}$. This is the case for [013], which acts as a twofold twin axis (Greek cross twin) and possibly also as a fourfold twin axis (Saint Andrew's twin): in the latter, the twin misfit $(0.493 \AA)$ is half of that of the former $(0.987 \AA)$.

The twin operations giving birth to the Saint Andrews cross twins are those belonging to the pseudo-cubic sublattice but not to the pseudo-tetragonal sublattice that corresponds to the
Greek cross twin. If we ignore the small deviation from the exact cubic metric of Mallard's pseudo-cube, the Saint Andrew's twins correspond to the trichromatic twin point group

$$
\left(\frac{4^{(2,1)}}{m} \overline{3}^{(3)} \frac{2^{(2,1)}}{m^{(2,1)}}\right)^{(3)}
$$

(Nespolo, 2004). The coset decomposition in Table 3 corresponds to the decomposition of this twin point group in terms of the dichromatic twin point group of the Greek cross twin.

\section{Conclusions}

The investigation of the reticular aspects of twins, despite its long history, is far from having said the last word. In the present study, we have shown that the cases of manifold twins were incorrectly treated both in terms of classification (zeroobliquity twins which nevertheless are TLQS twins) and of calculation of the twin index. The twin misfit is a more meaningful parameter to estimate the occurrence probability of a twin with respect to the twin obliquity, even if it is slightly more cumbersome to calculate, especially for manifold twins.

We wish to express our deep gratitude to Dr Bernd Souvigner (Radboud University, Nijmegen, The Netherlands) for fruitful discussions. The comments by two anonymous referees and by the Co-Editor Professor Manuel Perez-Mato are gratefully acknowledged. GF acknowledges the Italian Ministry of University and Scientific Research (MIUR) for financial support.

\section{References}

Alexander, V. D. (1989). Am. Mineral. 74, 610-619.

Bonnet, R. \& Durand, F. (1975). Philos. Mag. 32, 997-1006.

Cardoso, G. M. (1928). Z. Kristallogr. 66, 485-487.

Curien, H. \& Le Corre, Y. (1958). Bull. Soc. Fr. Minéral. Cristallogr. 81, 126-132.

Dana, E. S. (1876). Am. J. Sci. 11, 384-387.

Donnay, G. \& Donnay, J. D. H. (1974). Can. Mineral. 12, 422-425.

Donnay, J. D. H. \& Donnay, G. (1959). International Tables for X-ray Crystallography, Vol. III, Section 3.1.9. Birmingham: Kynoch Press.

Donnay, J. D. H. \& Donnay, G. (1983). Tschermaks Mineral. Petr. Mitt. 31, 1-15.

Ferraris, G., Makovicky, E. \& Merlino, S. (2004). Crystallography of Modular Materials. IUCr/Oxford University Press.

Friedel, G. (1904). Étude sur les Groupements Cristallins. Extract from Bulletin de la Société de l'Industrie Minérale, Quatrième Série, Tomes III et IV. Saint-Etienne: Société de l'Imprimerie Théolier J. Thomas et C.

Friedel, G. (1920). Bull. Soc. Fr. Minéral. 43, 246-295.

Friedel, G. (1922). Bull. Soc. Fr. Minéral. 45, 8-15.

Friedel, G. (1926). Leçons de Cristallographie. Nancy/Paris: BergerLevrault.

Griffen, D. T., Gosney, T. C. \& Revell Phillips, W. (1982). Am. Mineral. 67, 292-297.

Grimmer, H. \& Nespolo, M. (2006). Z. Kristallogr. 220, 28-50.

Hahn, T. \& Klapper, H. (2003). International Tables for Crystallography, Vol. D, edited by A. Authier, Section 3.3. Dordrecht: D. Reidel Publishing Company. 
Hurst, V., Donnay, J. D. H. \& Donnay, G. (1956). Mineral. Mag. 31, $145-163$.

Juurinen, A. (1956). Composition and Properties of Staurolite. Suomalaien Tiedeakat, Toimituksia, Helsinki.

Mügge, O. (1894). Neues Jahrb. Mineral. Geol. Paläontol. 1, 106-108. Náray-Szabó, I. (1929). Z. Kristallogr. 71, 103-116.

Náray-Szabó, I. \& Sasvári, K. (1958). Acta Cryst. 11, 862-865.
Nespolo, M. (2004). Z. Kristallogr. 219, 57-71.

Nespolo, M. \& Ferraris, G. (2000). Z. Kristallogr. 215, 77-81. Nespolo, M. \& Ferraris, G. (2003). Z. Kristallogr. 218, 178-181. Nespolo, M. \& Ferraris, G. (2005). Z. Kristallogr. 220, 317-323. Nespolo, M. \& Ferraris, G. (2006). Acta Cryst. A62, 336-349. Santoro, A. (1974). Acta Cryst. A30, 224-231.

Smith, J. V. (1968). Am. Mineral. 53, 1139-1155. 